\title{
Distribución nerviosa interna del músculo temporal humano: consideraciones anatómicas y quirúrgicas
}

\author{
Cristina Sanzana-Luengo ${ }^{1}$, Mauricio Sandoval T. ${ }^{1}$, Rodrigo Hernández Q. ${ }^{1,2}$, \\ Jorge Lemus E. ${ }^{1}$, Andrés Rosa-Valencia ${ }^{1}$, Rosa Córdova M. ${ }^{1}$ y Arnoldo Hernández C. ${ }^{1,2}$
}

\section{Internal nervous distribution of the human temporal muscle: anatomical and surgical considerations}

Introduction: Knowing in detail the inner innervation of the human temporal muscle allows to perform multiple surgical techniques and treatments of pathologies that involve the craniofacial territory. Although the internal innervation of the human temporal muscle based on direct microdissection has been described in the literature, the Sihler staining technique is an advantageous tool for anatomical study since it allows observing small nerve branches without losing its three-dimensional relationship with muscle fibers. Aim: To describe the nervous distribution within the human temporal muscle in cadavers by applying the Sihler method and analyzing its surgical anatomical association. Materials and Method: Eight previously dissected human temporal muscles were subjected to the Sihler staining method. Each one of the samples was observed under stereoscopic magnification and transillumination, finally for its description the muscle was divided into three regions. Results: The presence of three main nervous trunks was determined: the anterior deep temporal, the deep medium temporal and the posterior deep temporal, those that run from deep to superficial. In addition, collateral branches of lesser caliber of the posterior deep temporal nerve that in the form of an arc communicate the three regions of the muscle were observed. Conclusion: A common internal nervous distribution was described for the muscles studied in the three dimensions of space, useful knowledge to innovate in clinical-surgical therapies of the craniofacial territory.

Key words: temporal muscle; innervation; Sihler's staining.

\section{Resumen}

Introducción: Conocer en detalle la inervación interna del músculo temporal humano permite realizar múltiples técnicas quirúrgicas y tratamientos de patologías que involucran al territorio craneofacial. Si bien en la literatura se ha descrito la inervación interna del músculo temporal humano basado en microdisección directa, la técnica de tinción de Sihler es una herramienta ventajosa para el estudio anatómico ya que permite observar ramos nerviosos pequeños sin perder su relación tridimensional con las fibras musculares. Objetivo: Describir la distribución nerviosa al interior del músculo temporal humano en cadáveres al aplicar el método de Sihler y analizar su asociación anátomo quirúrgica. Materiales y Método: Ocho músculos temporales humanos previamente disecados fueron sometidos al método de tinción de Sihler. Cada una de las muestras se observó bajo lupa estereoscópica y transiluminación; finalmente para su descripción se dividió al músculo en tres regiones. Resultados: Se determinó la presencia de tres troncos nerviosos principales: el temporal profundo anterior, el temporal profundo medio y temporal profundo posterior, los que discurren de profundo a superficial. Además, se observaron ramos colaterales de menor calibre del nervio temporal profundo posterior que en forma de arco comunican las tres regiones del músculo. Conclusión: Se describió una distribución nerviosa interna común para los músculos estudiados en las tres dimensiones del espacio, conocimiento útil para innovar en terapias clínico-quirúrgicas del territorio craneofacial.

Palabras clave: músculo temporal; inervación; tinción de Sihler.
Instituto de Ciencias

Odontológicas, Facultad de Odontología. Universidad de Chile. Santiago, Chile.

2Departamento de Anatomía Humana y Medicina Legal, Facultad de Medicina. Universidad de Chile. Santiago, Chile.

Recibido el 30 de mayo de 2018 y aceptado para publicación el 5 de julio de 2018.

Correspondencia a: Dra. Cristina Sanzana-Luengo csanzana@ug.uchile.cl 


\section{Introducción}

El músculo temporal se utiliza para realizar colgajos que reconstruyan defectos en el territorio craneofacial luego de la resección de un tumor de la cavidad oral, base de cráneo u órbita, así como para el tratamiento de la parálisis facial ${ }^{1-5}$, dependiendo de su uso el colgajo de músculo temporal puede ser dinámico o estático ${ }^{6}$. En el primer caso, donde se busca la funcionalidad muscular es indispensable el conocimiento profundo del patrón de inervación como por ejemplo en la técnica quirúrgica de Labbé7.

El músculo temporal, se ubica en la fosa del mismo nombre y es descrito anatómicamente como una estructura ancha, plana, radiada, cubierta lateralmente por la fascia temporal ${ }^{8}$. Además, se caracteriza por ser altamente penado con una arquitectura compleja que sugiere diversidad de comportamiento en base a la disposición de sus fibras ${ }^{9}$. Sus acciones no pueden ser solamente determinadas a partir de su orientación general, ya que la evidencia ha demostrado la existencia de compartimentos neuromusculares inervados por un ramo nervioso primario que tienen una función e inervación selectiva al interior del músculo ${ }^{10,11}$. A la fecha, existe escasa evidencia que describa la distribución intramuscular de los ramos nerviosos del músculo temporal ${ }^{7}$, además, aún se desconoce el patrón de inervación de la totalidad del mismo, limitando su potencial uso.

La literatura clásica describe la inervación del músculo temporal a partir de la disección anatómica directa, estableciendo la existencia de tres porciones en un plano: la porción anterior, inervada por el nervio temporal profundo anterior (TPA); la porción media, por el nervio temporal profundo medio (TPM) y finalmente la porción posterior, por el nervio temporal profundo posterior (TPP $)^{12}$. Existen estudios actuales basados en microdisección directa que describen variantes del patrón de inervación y su compartimentalización ${ }^{7,13,14}$. Sin embargo, esta técnica anatómica imposibilita reconocer ramos nerviosos colaterales más pequeños, su posible arborización y, a su vez, causan la pérdida de las relaciones del nervio con las fibras musculares ${ }^{15}$.

La tinción de Sihler es una técnica anatómica creada por Charles Sihler en 1895 y que desde el año 1990 ha sido ampliamente utilizada en humanos. Su propósito es transparentar fibras musculares mientras tiñe de color violeta los troncos nerviosos a nivel intramuscular, lo que permite identificarlos fácilmente sin la disrupción estructural que provocaría la disección anatómica tradicional $1^{16}$.

El objetivo de este estudio es describir el patrón de inervación al interior del músculo temporal humano en cadáveres al aplicar el método de Sihler y analizar su asociación anatómica-quirúrgica.

\section{Materiales y Método}

Se realizó un estudio descriptivo de corte transversal. La muestra evaluada corresponde a ocho músculos temporales humanos fijados y conservados, el tamaño muestral se estableció por disponibilidad de cadáveres del Programa de Anatomía Humana y Medicina Legal de la Facultad de Medicina de la Universidad de Chile. Para la realización de este trabajo se contó con la debida autorización sanitaria y legal por parte de la Facultad de Medicina de la Universidad.

Los criterios de inclusión aplicados fueron: cadáveres de hombres y mujeres adultos con integridad estructural de la región temporal (tejidos lo suficientemente hidratados para permitir su disección) $\mathrm{y}$ ausencia de deformaciones externas evidentes.

Para la obtención de los músculos a estudiar se efectuó disección cadavérica de la región temporal, la que se describe brevemente: primero se realizó una incisión vertical desde la sutura sagital superiormente hasta $1,5 \mathrm{~cm}$ anterior al tragus inferiormente; se efectuó también una descarga posterior paralela al hélix hasta el proceso mastoides para facilitar el acceso. La incisión se extendió en profundidad hasta la fascia temporal, sin comprometerla. Luego, mediante disección roma y aguda, se disecó hacia posterior y hacia anterior el plano cutáneo y el sistema músculo-aponeurótico superficial en su conjunto. A continuación, los colgajos se rechazaron hacia posterior y anterior respectivamente a fin de acceder a la fascia temporal. Se efectuó una incisión hasta el plano óseo a lo largo de las inserciones periféricas de la fascia temporal. Con una legra se desinsertó el músculo hacia el tendón, decolando a través del plano subperióstico (Figura 1a). Finalmente, se seccionó el tendón de sus inserciones mandibulares, se identificó los troncos nerviosos principales y se retiró la pieza anatómica.

A los músculos temporales obtenidos se les retiró la fascia temporal para luego ser sometidos al método de tinción de Sihler, que sigue un protocolo subdividido en ocho etapas, las que, en su totalidad demoraron entre cuatro y cinco meses, dependiendo del grosor y tamaño de la pieza a estudiar ${ }^{15-17}$. Las etapas se resumen en la Tabla 1.

Una vez lograda la transparentación del músculo y la visualización de los nervios mediante transiluminación, las muestras se fotografiaron en sus caras 


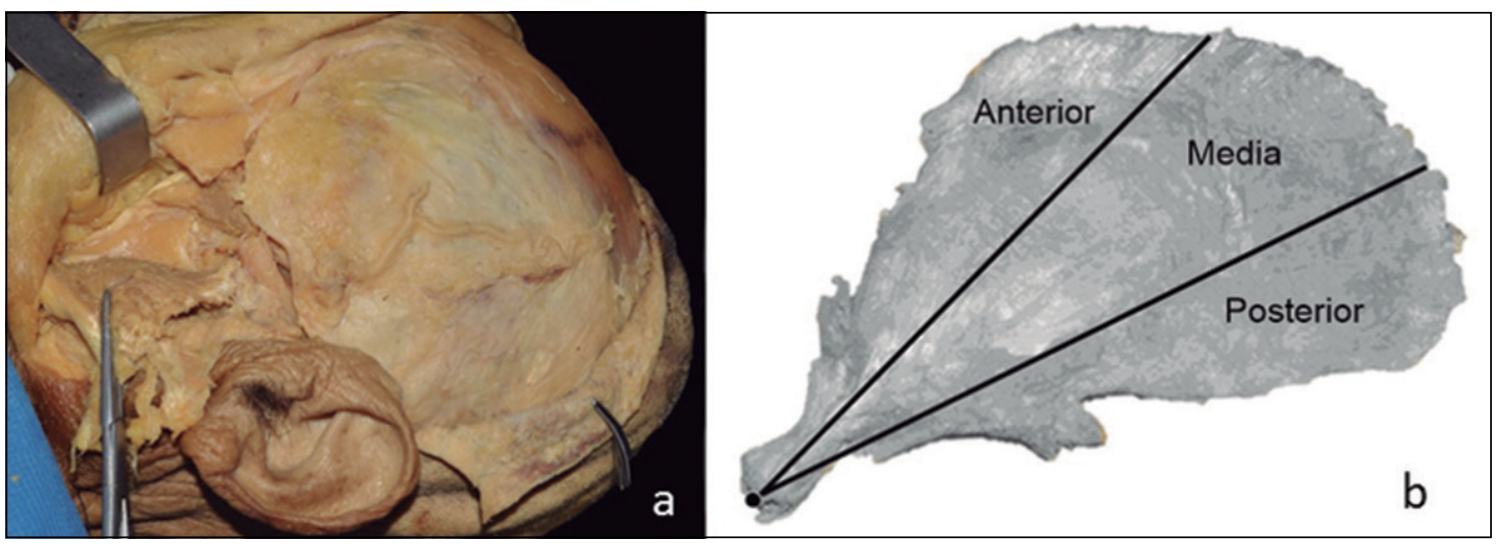

Figura 1. a) Visión lateral de la fosa temporal en un preparado cadavérico fijado luego del rechazo hacia anterior y posterior de estructuras superficiales y resección del arco cigomático; b) Visión lateral de músculo temporal dividido topográficamente en tres regiones equivalentes en el plano sagital.

Tabla 1. Etapas técnica de Sihler y cambios en el aspecto de la muestra de músculo temporal

\begin{tabular}{|c|c|c|c|c|}
\hline $\mathbf{N}^{\mathbf{o}}$ & Procedimiento & Solución & Tiempo & Aspecto \\
\hline 1 & Fijación & Formalina no neutralizada al $10 \%$ & 4 semanas & No hay cambios macroscópicos observados \\
\hline 2 & $\begin{array}{l}\text { Maceración y } \\
\text { despigmentación }\end{array}$ & $\mathrm{KOH}$ acuoso & 4 semanas & $\begin{array}{l}\text { Muestra comienza a blanquearse y los nervios pueden } \\
\text { verse como pequeñas fibras de color blanco }\end{array}$ \\
\hline 3 & Descalcificación & Solución Sihler I & 3 a 4 semanas & No hay cambios observados respecto a la etapa anterior \\
\hline 4 & Tinción & Solución Sihler II & 4 semanas & $\begin{array}{l}\text { Todas las estructuras nerviosas, serán observadas de un } \\
\text { color azul violeta oscuro }\end{array}$ \\
\hline 5 & Desteñido & Solución Sihler I & 4 horas & $\begin{array}{l}\text { Las muestras deben ser observadas por transiluminación, } \\
\text { esta etapa debe ser detenida cuando los nervios adquieran } \\
\text { un color azul oscuro o violeta }\end{array}$ \\
\hline 6 & Neutralización & Carbonato de litio & 1 a 2 horas & $\begin{array}{l}\text { El procedimiento debe ser detenido cuando el color de los } \\
\text { nervios pase de violeta a azul oscuro }\end{array}$ \\
\hline 7 & Aclaramiento & $\begin{array}{l}\text { Glicerina acuosa a distintas con- } \\
\text { centraciones }\end{array}$ & 3 a 4 días & $\begin{array}{l}\text { Esta etapa debe ser detenida cuando los nervios pequeños } \\
\text { puedan ser observados bajo el microscopio de disección }\end{array}$ \\
\hline 8 & Transparencia & Glicerina con timol & & \\
\hline
\end{tabular}

medial y lateral con una cámara Nikon modelo D3100 (Nikon Corp., Tokyo, Japón) y un lente macro Raynox modelo Der 250 (Yoshida Industry Co., Tokyo, Japón) con apertura F8, ISO 100. En una sala oscura, se posicionó el músculo a estudiar sobre un negatoscopio de luz blanca dispuesto de forma horizontal con una regla de $20 \mathrm{~cm}$ sobre su superficie. La cámara se ubicó en un soporte horizontal a treinta centímetros de la superficie del negatoscopio. En el caso de las estructuras nerviosas más finas, estas se visualizaron y fotografiaron bajo lupa estereoscópica Zeiss Stemi DV4 (Industrial BioSciences, Jena, Germany) a distintos aumentos.

Para realizar el análisis descriptivo de las imágenes obtenidas se manejaron en grado de saturación, luz y nitidez, mediante el software Adobe Photoshop CS4 (Adobe Systems incorporated, San José, California, EE. UU.) y luego se dividieron topográficamente en tres regiones equivalentes en el plano sagital: anterior, media y posterior, trazando dos líneas con origen en el vértice del tendón hacia el borde superior del músculo (Figura $1 \mathrm{~b}$ ). Una vez realizada la división se registraron las siguientes variables: Número de troncos principales, distribución de cada uno de estos troncos en las regiones topográficas previamente establecidas, presencia y distribución topográfica de los ramos colaterales, comunicación de los ramos colaterales. Finalmente, en base a los resultados obtenidos, se propuso un esquema del patrón de inervación interno del músculo temporal. 


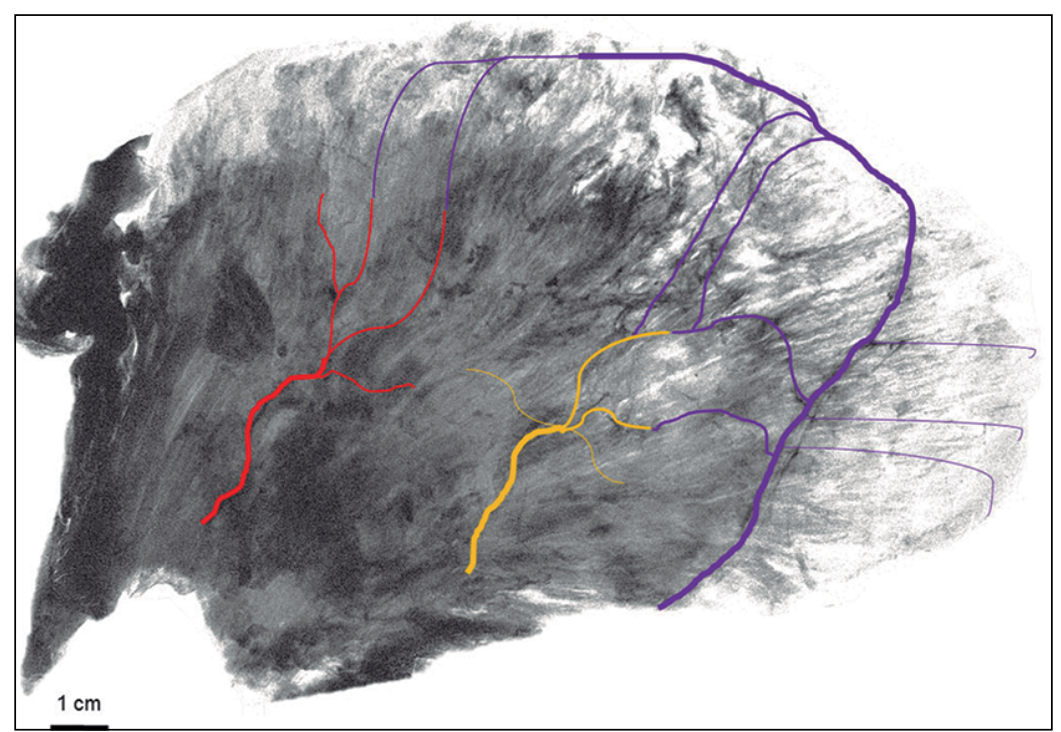

Figura 2. Esquema propuesto del patrón de inervación interno del músculo temporal en el plano sagital, en color violeta se observa nervio TPP y sus comunicaciones, en color amarillo se observa TPM, en color rojo se observa distribución del nervio TPA.

\section{Resultados}

En las 8 muestras estudiadas se observó un patrón de inervación interno que se describe a continuación.

El músculo temporal está inervado por tres troncos nerviosos principales TPA, TPM y TPP, los que ingresan por la cara medial del músculo y se distribuyen en las regiones topográficas anterior, media y posterior, respectivamente (Figura 2).

El nervio TPA accede al músculo temporal por la porción inferior de la región anterior, área que se caracteriza por ser altamente tendinosa y la de mayor espesor. Este discurre de profundo a superficial penetrando las fibras musculares en el tercio medio, la dirección del tronco TPA es de anterior a posterior y de inferior a superior recorriendo de forma oblicua la región topográfica anterior, sus ramos colaterales superficiales se comunican con los ramos colaterales de los nervios TPM y TPP (Figura 3A).

El nervio TPM accede al músculo temporal por inferior de la región media. El tronco principal penetra de profundo a superficial y discurre de forma paralela a las fibras musculares, sus ramos colaterales en forma de arco se originan en la unión del tercio superior con el medio y se dirigen hacia posterior hasta unirse con el nervio TPP, de estos ramos comunicantes surgen pequeños ramos nerviosos que ascienden y descienden paralelo a las fibras, de este modo logran inervar una amplia superficie del músculo (Figura 3B).

El nervio TPP es el de más fácil visualización de los tres troncos y accede al músculo temporal por el borde inferior de la región posterior. El tronco principal discurre en la porción profunda del músculo de forma oblicua a las fibras musculares con una dirección que va de anterior a posterior y de inferior a superior. De este tronco principal en el tercio medio se desprenden ramos colaterales hacia anterior y posterior. Cuando el tronco principal llega a la porción superior de la región posterior cambia su dirección de posterior hacia anterior y adquiere forma de arco del cual emergen ramos colaterales superficiales que comunican los tres troncos nerviosos TPA, TPM, TPP (Figura 3C).

\section{Discusión}

En la literatura existen estudios que mediante el uso de microdisección anatómica directa describen el patrón de inervación intramuscular del temporal ${ }^{13,14,18,19}$. En ellos se identificaron tres áreas: anterior, media y posterior, inervadas por los nervios TPA, TPM y TPP respectivamente. A diferencia de Chang et al. ${ }^{7}$, quienes al utilizar la técnica de microdisección y posterior reconstrucción tridimensional describieron cinco ramos nerviosos que inervan regiones independientes: tres superiores por los nervios TPA, TPM, TPP y dos inferiores por el nervio bucal y el nervio mesentérico ${ }^{6}$. Sólo dos estudios en el mundo han aplicado el método de Sihler en el músculo temporal humano, en uno de ellos se realizó microdisección posterior a la tinción y se describe la existencia de un tronco nervioso principal que se dividió en dos ramos uno anterior y otro posterior en estrecha relación a la arteria temporal profunda ${ }^{20}$.

Se debe mencionar que los métodos utilizados en estos estudios tienen las limitaciones descritas anteriormente producto de la microdisección.

Los resultados del presente estudio concuerdan con la literatura que describe tres troncos nerviosos principales TPA, TPM y TPP, que inervan tres regiones: anterior, media y posterior respectivamente. Además, en cuanto a su distribución tridimensional, se observó que estos transcurren de profundo a superficial penetrando las fibras musculares, lo que reafirma los hallazgos de Choi et $\mathrm{al}^{21}$.

Se debe destacar que a través de nuestro estudio fue posible observar ramos de menor calibre provenientes del nervio TPP y sus comunicaciones en forma de arco con los ramos colaterales de los nervios TPM y TPA. 


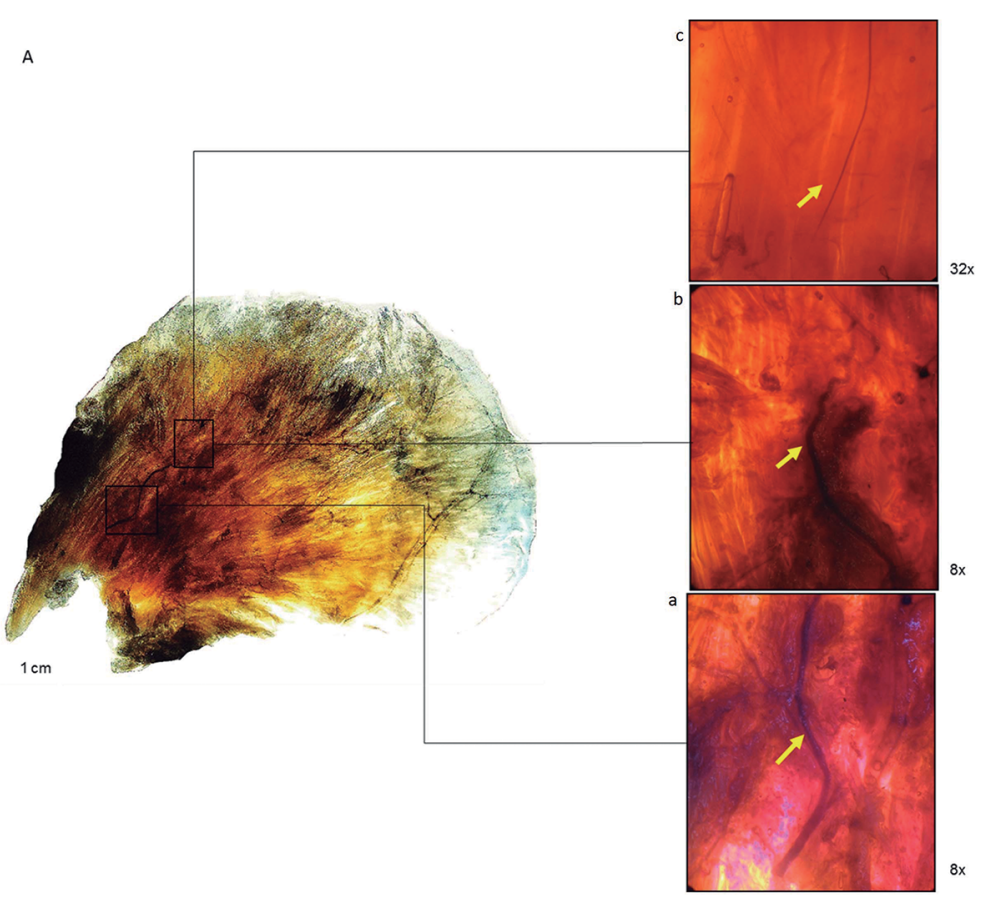

B
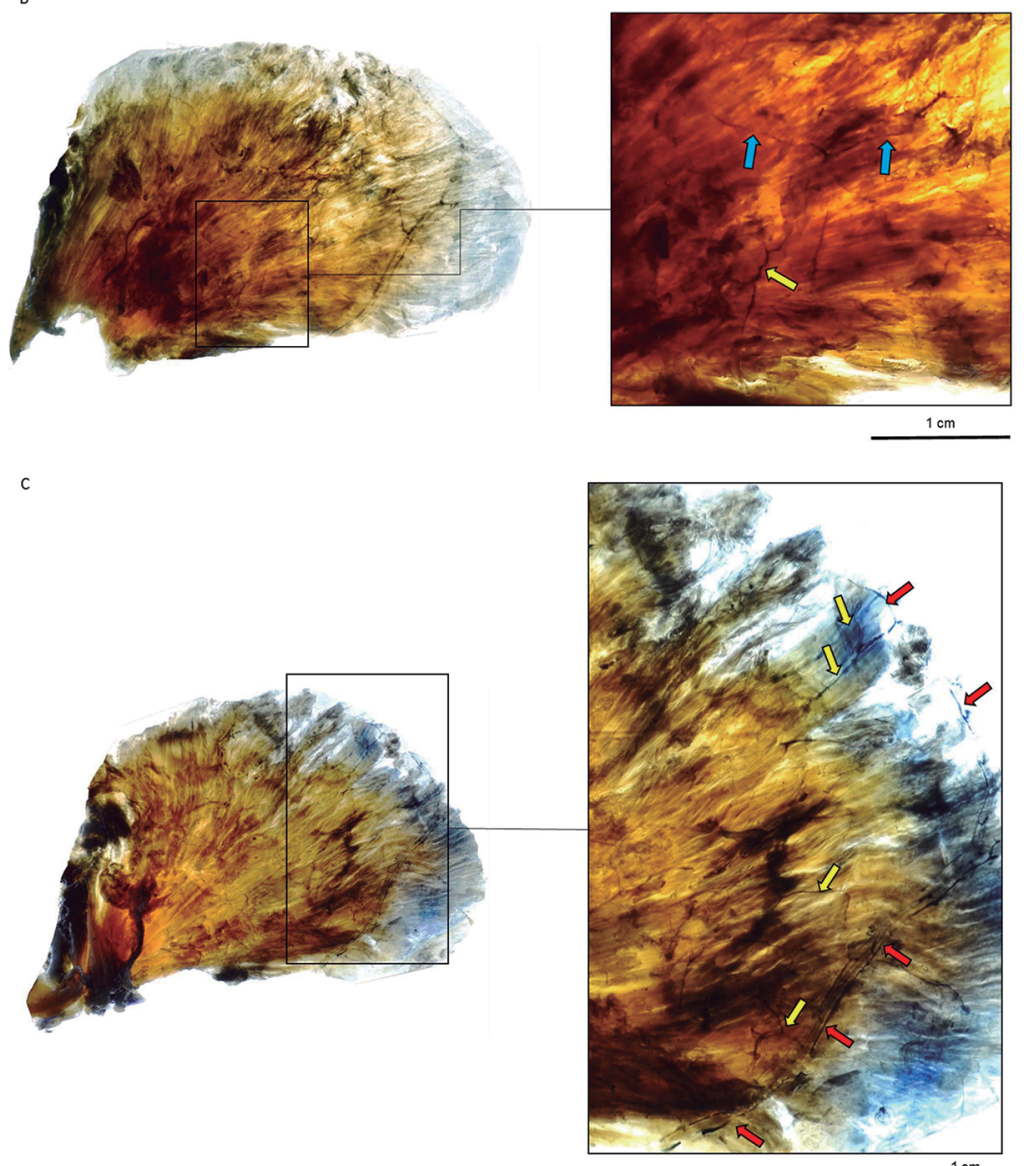

Figura 3 A. a) Flecha amarilla indica tronco nervioso TPA en cara medial del músculo; b) microscopía de la cara medial de músculo, flecha amarilla muestra nervio TPA penetrando fibras musculares; c) microscopía cara lateral músculo, flecha amarilla indica fibras nerviosas del TPA. B: Fotografía cara lateral del músculo temporal, flecha amarilla muestra tronco principal de TPM, flechas azules muestran arborización de TPM. C: Fotografía cara lateral del músculo, flechas rojas muestran tronco principal del nervio TPP y flechas amarillas muestran sus ramos comunicantes. 
Estos resultados obtenidos confirman que cada una de las regiones del temporal está inervada intramuscularmente por un tronco principal distinto (TPA, TPM y TPP), lo que sugiere la conformación de tres compartimentos funcionalmente independientes en concordancia con la literatura ${ }^{7}$. Esta compartimentalización anatómica y probablemente funcional, nos hace pensar que existe una activación independiente de cada una de las tres porciones del músculo, ya que todos tienen un tronco principal que preservaría su función. De esta forma se podría innovar en el diseño de colgajos funcionales y unidades músculo tendón utilizadas en la técnica de reanimación facial.

De manera adicional, nuestros resultados colaboran aportando información anatómica del músculo temporal para realizar otros tratamientos quirúrgicos y no quirúrgicos como es el caso de trastornos temporomandibulares, la hipertrofia del músculo temporal y distonía oromandibular, cuyos enfoques terapéuticos se basan en el bloqueo motor del músculo temporal ${ }^{21-23}$ o mediante denervación selectiva del nervio TPA ${ }^{24}$.

Finalmente, este estudio permitió describir el patrón de inervación interno del músculo temporal humano mediante la tinción de Sihler destacando su distribución de profundo a superficial, sus ramos nerviosos colaterales de menor calibre y sus comunicaciones sólo una vez reportadas anteriormente en la literatura. Si bien esta información aporta al conocimiento anatómico-quirúgico es necesario complementar con estudios electromiográficos para confirmar las sugerencias realizadas en este artículo.

\section{Agradecimientos}

Se agradece a la Dra. Viviana Toro y al equipo de funcionarios y docentes del Departamento de Anatomía Humana y Medicina Legal de la Universidad de Chile.

\section{Responsabilidades éticas}

Las imágenes e información empleadas en este artículo provienen de disecciones de material cadavérico pertenecientes al Departamento de Anatomía y Medicina Legal de la Universidad de Chile.

Protección de personas y animales: Los autores declaran que los procedimientos seguidos se conformaron a las normas éticas y de acuerdo con la Asociación Médica Mundial y la Declaración de Helsinki.

Confidencialidad de los datos: Los autores declaran que en este artículo no aparecen datos de pacientes.

Conflicto de interés: Los autores del presente artículo declaran no tener conflictos de interés.

Fuente de apoyo académico: Proyecto PRIODO N $\mathrm{N}^{\circ} 2015 / 05$.

Fuente de financiamiento: En cuanto a las fuentes de apoyo financiero no existieron tales.

\section{Referencias}

1. Zubillaga I, Sánchez G, García I, Montalvo J. Utilización del colgajo de músculo temporal en cirugía reconstructiva maxilofacial. Revisión de 104 casos. Rev Esp Cirug Oral y Maxilofac. 2004;26:228-37.

2. Vidal C, Lanzarini S, Bancaliari D. Cirugía de la asimetría en la parálisis facial periférica. Rev Chil Cir. 2005;57:155-9.

3. Ahmad S, Warraich RA, Abid H, Ali S. The Versatility of Temporalis Muscle Flap in Reconstruction of Maxillofacial Region Defects. Annals of KEMC. 2009;15:2730.

4. Lam D, Carlson ER. The temporalis muscle flap and temporoparietal fascial flap. Oral Maxillofacial Surg Clin N Am. 2014;26:359-69.

5. Campos O, Gutiérrez T. Parálisis facial permanente: tratamiento quirúrgico en base a la técnica de Labbé. Rev Chil Cir. [edición electrónica] 2006 [citado el 25 de abril de 2018];58(2):159-64. Disponible en: https://scielo.conicyt.cl/ scielo.php?script=sci_arttext\&pid=S071840262006000200015\&lng=es. http://dx.doi.org/10.4067/S071840262006000200015

6. Veyssiere A, Rod J, Leprovost N, Caillot A, Labbé D, Gerdom A, et al. Split temporalis muscle flap anatomy, vascularization and clinical applications. Surg Radiol Anat. 2013;35:573-8.

7. Chang Y, Cantelmi D, Wisco JJ, Fattah A, Hannam A, Agur A. Evidence for the functional compartmentalization of the temporalis muscle: A 3-dimensional study of innervation. J Oral Maxillofac Surg. 2013;71:1170-7.

8. Gaudy JF, Zouaoui A, Bri P, Charrier JL, Laison F. Functional anatomy of the human temporal muscle. Surg Radiol Anat. 2001;23:389-98.

9. Hannam AG, McMillan AS. Internal organization in the human jaw muscles. Crit Rev Oral Biol Med. 1994;5:55-89.

10. Van Eijden TM, Koolstra JH, Brugman P. Three-dimensional structure of the human temporalis muscle. Anat Rec. 1996;246:565-72.

11. English AW, Wolf SL, Segal RL. Compartmentalization of muscles and their motor nuclei: The partitioning hypothesis. Phys Ther. 1993;73:857-67. 
12. Rouviere H, Delmas A. Anatomía humana descriptiva, topográfica y funcional. Tomo I. $11^{\mathrm{a}}$ ed. Barcelona: Masson SA; 2005. p. 290-92.

13. Shimokawa T, Akita K, Soma K, Sato T. Innervation analysis of the small muscle bundles attached to the temporalis: truly new muscles or merely derivatives of the temporalis? Surg Radiol Anat. 1998;20:329-34.

14. Burggasser G, Happak W, Gruber H, Freilinger G. The temporalis: blood supply and innervation. Plast Reconstr Surg. 2002;109:1862-69.

15. Mu L, Sanders I. Sihler's whole mount nerve staining technique: A review. Biotech Histochem. 2010;85:19-42.

16. Won SY, Kim DH, Yang HM, Park JT, Kwak HH, Hu KS, et al. Clinical and anatomical approach using Sihler's staining technique (whole mount nerve stain). Anat Cell Biol. 2011;44:1-7.

17. Yang HM, Won SY, Lee JG, Han SH, Kim HJ, Hu KS. Sihler-stain study of buccal nerve distribution and its clinical implications. Oral Surg Oral Med Oral Pathol Oral Radiol. 2012;113:334-9.

18. Geers C, Nyssen-Behets C, Cosnard $\mathrm{G}$, Lengelé $\mathrm{B}$. The deep belly of the temporalis muscle: an anatomical, histological and MRI study. Surg Radiol Anat. 2005;27:184-91.

19. Karagoz H, Ozturk S, Malkoc I, Diyarbakir S, Demirkan F. Anatomy of the anterior deep temporal nerve: Implications for neurotization in blinking restoration in facial paralysis. Ann Plast Surg 2015;75:316-18

20. Ziccardi VB, Mu L, Schneider RE, Sanders I. Innervation pattern of the temporalis muscle. J Craniofac Surg 1998;9:185-9.
21. Schiffman E, Ohrbach R, Truelove E, Look J, Anderson G, Goulet JP, et al. Diagnostic Criteria for Temporomandibular Disorders (DC/TMD) for Clinical and Research Applications: Recommendations of the International RDC/TMD Consortium Network* and Orofacial Pain Special Interest Group $\dagger$. J Oral Facial Pain Headache. 2014;28:6-27.

22. Alter KE, Wilson NA, editores. Botulinum Neurotoxin Injection Manual. New York: Demos Medical Publishing; 2014:69-76.

23. Choi YJ, Lee WJ, Lee HJ, Lee KW, Kim HJ, Hu KS. Effective botulinum toxin injection guide for treatment of temporal headache. Toxins (Basel). 2016;8:265.

24. Hwang K, Cho HJ, Chung IH. Innervation of the temporalis muscle for selective electrical denervation. J Craniofac Surg. 2004; 15:352-7. 